\title{
Buch Anzeigen - Besprechungen und Notizen
}

Dr. A, Blaschko. Syphilis und Prostitution vom Standpunkte der öffent-liclien

Oesundheitspfleg'e. Berlin, Verlag von S. Karger.

Durch dieses neuesto Werk des auf deni Gebiete der Prophylaxe der vene-i $1 / 8$ chen Krankheiten und des Prostitutionswesens hekannten Autors, welches, wie 1/8us dem Vorworte und aus dem ersten in klarer Weise die verschiedenen venerischen Affectionen und ihre Folg-ezustiinde behandelnden Kapitel hervorgebt, nicht nur für Mediziner, sondern auch für weitere der Hygieine der venerischen Krank-lieiten nahestehende Kreise bestitnmt ist, hat sich der Verfasser ein grosses Ver-

$-380-$

dienst erworben. Das Buck kat durck die zaklreicken lirterariscken Belege und Statistiken, zumal auf deni Gebiete des Militärsanitätswesens, sowie durck eigene statistiscke Erhebungen nock besonderen Wert.

Eine eingekendere Bespreckung des mit Reglernents, Medicinaledikten polizei-licben Vorsckriften, Tkesen der Prof. Thiry (Brüssel) und Kaposi (Wien) vom X. internat. medicin. Kongress zu Berlin etc. etc. entkaltenden Anbange versekenen Werkes kann.bei dem grossen Umfange desselben (202 Seiten) nickt kier Platz finden, nur Einzelnes möge kervorgekoben werden. Zunäckst ist es dankbar zu begrüssen, dass Blaschko auf die grossen, nock allgemein untersckätzten, Gefabren der Gonorrkoe hinweist. Aus zaklreicken fremden und eigenen Statistiken kommt Blaschko zu dem Resume, dass 60 bis 70 pOt. aller Geschlechtskranken Tripperkranke sind, wäkrend nur 20 bis 30 pCt, der Gescklecktskranken syphilitisch sind. Auck der Sypkilis gegenüber untersckätzt Blaschko nickt die Bedeutung der Gonorrkoe, was folgender Satz zur Genüge beweist: „Welcke der beiden Krankkeiten in der Eke mekr Sckaden stiftet, ist sckwer zu sagen" (pag. 53).

Unter diesen Umständen ist es auck erklärlich, dass sick der V"erfasser - selbst unter Berücksicktigung der neuerdings von Bröse erkobenen Einsprücbo - der Neisser schen Forderung der mikroskopiscken Untersuckung des Urethral-und Cerical-Secrets auf Gonokokken bei Prostituirten anscbliesst.

$\mathrm{Zu}$ weit gebt aber Blaschko in der Verfecktung seines scbon früker mebr-fack bekannt gegebenen Standpunktes als Bordellgegner.

So gesckickt seine Deduktionen und so günstig die von ikm zum Beweise kerbeigezogenen kritiscken Belege sind, so vermag er nack Ansickt des Referenten nicktsdestoweniger die berecbtigte Stellung der Gegner nickt zu erscküttern.

Die Bekauptung Blaschko's, ,dass die sanitäre Kontrolle der wirklick gewerbsmässigen Puellen, sowie sie keute geübt wird, nur emeu recbt mangelkaften Sckutz gewährt" (pag. 86), wird aber obne Zweifel jeder auf diesem Gebiete Erfakrene voll und ganz mit untersckreiben, wenn dieses auck weniger wohl an der Art der Untersuckung als viel·mehr zum grössten Teile an der Seltenbeit der vorgenommenen Untersuckung liegen diirfte.

Interessant ist in dieser Richtung die von Blaschko zusammengestellte Untersuckungsskala der Prostituirten in den versckiedensten Städten (pag. 68). Daraus ergiebt sick, dass nur in Hannover 
4 Mai wöckentlick untersuckt wird, in alien anderen Städten Deutscblands köckstens 1-2 Mai wöckentlick.

Nickt minder bemerkenswert sind die vom Verfasser zum Teil wörtlick an-gefübrten Berickte der versckiedensten Gewerberäte Deutscblands, die sämmtlick anerkennen, dass gerade der sckleckte Verdienst zaklreicke Arbeiterinnen der Prostitution in die Arrne treibt.

Mit Nackdruck und unter Anfükrung eklatanter Beispiele weudet sick Blaschko gegen den $\S 6 \mathrm{a}$ des Krankenkassengesetzes, wonack Personen, die durck gescklecktlicke Aussckweifungen erkrankt sind, das Krankengeld entzogen wurde, mit Recbt verlangt er die Gleickstellung der venerisck Erkrankten mit anderen Kranken, wendet er sick gegen gewisse sanitätspolizeilicke Zwangsmassregeln (obligatoriscke Meldepílicbt, obligatoriscbe Untersuckungen der Arbeiter grösserer industrieller Etablissements), und weist er die Undurckfükrbarkeit der Lex Heinze nack: kurz, das Buck Blaschko's entkält ausser einer guten Uebersicht über den gegenwärtigen Stand der Prostitutionsfrage so viel Wissenswertes und Anregendes. dass es dem Arzte aufs Beste empfoklen werden kaim.

Dr. Neuberger, Nürnberg.

Dr. F. M. Oberlaender, Lehrbuch der Urethroskopie. Mit 9 bunten Tafeln und 21 Abbildungen im Texte. Leipzig, Verlag von GeorgTkieme, 1893. Besprocken von Earn. Saalfeld (Berlin). Nackdem es vermöge der ausserordentlicben Fortscbritte in der medici-niscken Technik gelungen war, Körperköklen vermittelst geeigneter Beleucktungs-instrumente dem Auge zugänglick zu macken, ist in den letzten Jakren einem speciellen, Erkrankungen sebr käufig ausgesctzten Organ, der Harnröbre, be-sondere Aufmerksamkeit bozüglich der Beleucbtung geschenkt worden. Der tkerapeutiscke Pessimismus und Nihilismus, der noch bis vor nickt zu langer Zeit

$-381-$

hinsichtlich der chronischen Gonorrhoe bestand, ist infolg·e der Entdeckung des Gonococcus, infolg $\cdot e$ der Klarstellung der pathologischen Verhältnisse gewichen und an seine Stelle teilweise eine Polypragmasie getreten, die allerdings nicht selten über das erstrebenswerte Ziel hinausging. Jedenfalls stehen wir jetzt der chronischen Gonorhoe nicht mehr so machtlos gegenüber wie friiher, und eine jede Methode, die eine Vervollkommnung unserer Kenntnisse berbeizuführen im-stande ist, muss dankbar begrüsst werden. Wenn auch die Meinungen über den Wert der Endoskopie der Harnröhe - ganz allgemein - noch keineswegs in jeder Hinsicht übereinstimmen, so bricbt sich doch die Meinung von der Wich-tigkeit derselben fur zahlreiche Entdeckungen immer mehr Bahn, und besonders war es Oberlaenãer, der in der letzten Zeit gemeinschaftlich mit Kollmann die Methode der Beleuchtung und der endoskopischen Therapie der Harnröhre weiter auszuarbeíten bemüht war. Nachdem nunmehr fünf Jahre vergangen, seitdem Oberlaenãer seine urethroskopischen Beobachtungen in einer ausführliclien Arbeit in der Vierteljahresschrift für Dermatologie und Syphilis niedergelegt, hat sich der Verf. veranlasst gesehen, die inzwischen neu gesammelten Erfahrungen und Ergänzungen seiner früheren Ansehauungen in einem Lehrbuche der Urethroskopie zu veröffentlichen.

Oberlaenãer benutzt bei seinem Elektro- Urethroskop im Пegensatz zu vielen anderen Endoskopíkem, die refiektiertes Licht anwenden, die direkte Be-leuchtung der erkrankten Stelle. Er äussert sich hierüber mit folgenden Worten: .,Das Elektro-Urethroskop ist durch seinen Vorzug der Einführung der Licht-quelle in die Harnröhre bis an Ort und Stelle etwas complicierter und difficiler in der Handhabung, andererseits hat es sich aber durch Jahrzehnte (? Ref.) lange Versuche und Arbeiten mit den anderen Instrumenten herausgestellt, dass man die für Diagnose und Therapie nötigen pathologisch-anatonûschen Details mit der Beleuchtung 
durch refiectiertes Licht nicht genügend erkennen kann. In-dessen verhält es sich mit dem Urethroskop wie mit jedem anderen Instruniente, wer mit einem anderen die Untersuchung gelernt hat und dasselbe .Tahre laug mit scheinbar genügendem Erfolg benutzt, wird schwer von der zu geringen Leistungsfiihigkeit derselben zu überzeugen sein." Alsdann führt uns der Verf. die von ihm und Kollmann benutzten Instrumente in ihren Einzelheiten vor und erläutert dabei ausführlich die Gebrauchsregeln für das Etektro-Urethroskop und die Technik der Untersuchung, wobei besonders die Cocainisierung der Harnröhre eingehend erörtert wird. Als eine der wichtigsten Regeln, die allerdings eigent-lich selhstverständlich ist, aber nicht oft genug wiederholt werden kann, sieht Verf. es an, die Sohleimhaut stets frei und rein von jeder Auflagerung zu machen, da man sonst unklare Bilder enthält. In den beiden folgenden Kapiteln werden wir mit der Endoskopie der normalon Harnröhe vom Orificium externum bis zum Bulbusende bekannt gemacht. Dann folgen die pathologischen Ver- $\Gamma$ tnderungen der Epitheldecke und der Drüsen und die histologischon Veränderungen der Urethralschleimhaut, speciell bei chronischer Gonorrhoe. Der nun folgende Toil ist der Endoskopie der Entzündungsformen des Mucosagewebes gewidmet. Verf. behandelt hier die verschiedenen Infiltratformen, die weichen, die In-íiltrationsformen von geiingerer und mittlcrer Mächtigkeit und dann die harten Infiltratformen stärkster Ausbildung, die Stricturen der Harnröhre. Das zwölfte Kapitel erörtert die Endoskopie bei Erkrankungen der hinteren Harnröhre, die naturgemass nur seltener und nur unter bestimmten $\backslash$ Toraussetzungen auszuführen ist. Ûarauf folgt die überaus wichtige Endoskopie bei den Geschwülsten der Harnröhe und im Schlusskapitel werden wir über die bisher mit Unrecht zu sehr vernachlässigte, keineswegs Schwierigkeiten darbietende urethroskopische Untersuchung der weiblichen Harnröhre belehrt. Zur besseren Erläuterung der Auseinandorsetzungen dienen neben mehreren Abbildnngen im Texte neun farbige, sehr sorgfältig ausgeführte Tafeln und eine Reihe von interessanten Krankengeschichten. Das Buch macht es möglich, sich über die einzelnen Krankheitsforraen genau zu informieren und wird es seinen Zweck, als Lehrbuch der Urethroskopie zu dienen, gewiss erfüllen. Die Folgezeit wird lehren, ob die oben citierte Anschauung des $\backslash \Gamma$ erf.'s, dass die Lichtquelle an den Ort der Erkrankung - gebracht, vor dem vor dem reflek-tierten Licht in der vorderen Harnröhre den Vorzug verdient. zu Rechte besteht. Doch werden hier erst weitere, noch zu sammelnde Erfahrungen eine Entscheidung bringen können. - Die Ansstattung des Buches ist vorzüglich,

$-382$

und der relativ geringe Preis fiir ein mit so vielen colorierten und einfachen Abbildungen versehenes Werk macht die Ansehaffung jedem Praktiker möglich.

Dr. G. Posner, Privafcdocent an der Uníversität Berlin, Diagnostik der Harnkrankheiten. Zehn Vorlesungen zur Binführung in die Pathologie der Harnwege. Mit 42 Abbildungen und einem symptomatologischen Anhang. Berlin 1894. Verlag von August Hirschwald. Besproehen von E. Saalfeld (Berlin). Während das Oberländerscho Werk einen ganz circumscripten Absclmitt aus der Lehre der Krankheiten der Harnorgane nmfasst ', bietet P. eine Diagnostik über das gesamte Gebiet der Harnkrankheiten. In gedrängter Kiirze und klarer Darstellungsweise führt alle einzelnen Facta vor, die fiir die Diagnose der Harnkrankheiten überhaupt in Betracht kommen. Die einfachen wie die minutiösen Untersuchungsmethoden werden dargestellt. Zuerst werden wir über die Beziehungen der Harnleiden zum Gesamtorganismus in Kenntnis gesetzt, Alsdann folgen die Harnröhrenausflüsse, über deren Wesen und Be-deutung noch vielfach falsche Anschauungen und V"erwirrung herrschen, und die richtig zu deuten, fiir den Arzt von allergrösstem Werte ist. Bei den nicht minder wichtigen Störungen der Harnentleeriitig führt uns 
der Verf. sämtliche ätiologischen Momente vor und berücksichtigt auch alle nicht in den Harnorganen selbst gelegenen Veranlassungen der Störungen der Harnentleerung. Die Harnuntersuchung wird in zwei gesonderten Kapiteln abgehandelt, u. s. w. werden zuerst die physikalischen und ohemischen Methoden und alsdann in einem grösseren Abschnitte die leider noch nicht allseitig genügend gevvürdigten mikro-skopischen ßefunde abgehandelt. Das sechste Kapitel betrifft die Inspection und Palpation. Kurz kommen bier alle einschlägigen Verhältnisse zur Sprache, und die verschiedenen DifTerentialdiagnosen werden hervorgehoben. In den nächsten vier Kapitein wird die Untersuchung der Harnröhre und der Blase besproehen. Zuerst werden wir über die Sondenuntersuchung resp. den Kathete-rismus und alsdann über die optischen Untersuchungsmethoden, die Endoskopie und Kystoskopie belehrt. Den Schluss des Werkes bildet ein Anhang,' der übersichtlich und prägnant eine Symptomatologie der gewöhnlichen Erkrankungen der Harnwege darstellt.

Zur Empfehlung des Buclies etwas hinzuzuíügen. halten wir nach dem Gesagten für überflüssig. Wenn wir einem Wunsche Ausdruck geben dürfen, so ist es der, dass der Verf. der Diagnostic recht bald eine g'leich lehrreiche Therapie der Harnkrankheiten folgen lassen möge.

Besprechungen und Notizen.

Dr. Xaase, Yorstellung · zweiei* Fälle vou mulü $\mu$ le« Keloiden. (Verb, der freieu

Verein. d. Chirurg. Berl. Jahrg. IV, S. 19.)

Ein lljähriger kräftiger Knabe leidet seit 41/2 Jahren an multipeln

spontanen Keloiden der Finger und Zehen. Nach Entfernung der Ge-

schwülste wuchern dieselben wieder; im letzten Jahre eutstanden Contracturen

der Finger. Bei Druck und Kälte siud die Geschwülste empfindlich. Wegen

Beschwerden beim Gehen wurde die zweite und dritte Zehe entfernt. Die

Wunden heilten gut. Die Contracturen sind durch diffuse derbe Infiltration en und Schrumpfungen der Haut und des Unterhautgewebes bedingt. Die Ge schwülste an den Fingern und Zehen stehen einauder gegeniiber wie Infectionsgeschwülste; jedoch hat sich mikroskopisch niclits gefunden, was für die parasititre Entstehung der Krankheit sprache. George Meyer (Berlin).

Dr. M. Mendelsohn, Harnrölireiistrictur. (Sep.-Abdr. a. Diagn. Lex. f. prakt Aerzte v. Bumm und Sehnirer.)

„Stricturen der Harnröhre sind Zustände derselben. in welcher die Harnröhre, an einer oder an mehreren Stellen ihres Verlaufes ihre normale Enthalt-qarkeit, wie sie bei jedor Harnentleerung stattfinden müsst»e, in grösserem oder

$-383-$

gßringerem Grade verloren hat." Ursache der Stricturen sind Traumen ùn'd langdauernde

F1/8tzündungen der Harnröhre, besonders Gonorrhoe. Die Gestalt der Stricturen ist sehr verschieden; ihro Zahl ist nur ausnahmsweise $\theta$ ine mehr-fache. Der Grad der Verengerung ist gleichfalls verschieden. Die traumatischen Stricturen sitzen meistens am Bulbus und dessen Gegend; die blenorrhagischen Stricturen flnden sich besonders im Uebergang der Pars bulbosa zur Pars mem-branacea. Die Erscheinungen der Harnröhrenverengerung betreffen zunächst die Entleerung des Samens, dann die des Harns. Es entsteht Enurese, Harnver-haltung und schliesslich Veränderung des Harns durch Fortschreiten des Krank-heitsvorganges nach höher gelegenen Abschnitten. Betastung und Sondenuzter-suchung sind zur Erkennung der Stricturen von Bedeutung, weniger wichtig ist die endoskopische Untersuchung. Es wird zum Schluss der Verlauf der Er-krankung und deren Differentialdiagnuse in Kiirze geschildert.

George Meyer (Berlin). 
Dr. 8. Ehrmann, Ueber Sykosis und Folliculitis. ("Wiener Medicinische Presse 1893. No. 9 und 10.))

Die Sykosis ist eine auf behaarten Stellen (nicht nur am Barte) localisierte. folliculäre Eiterung. Der Name Folliculitis bezeichnet gleichzeitig die Eingangspforte der Krankheitserreger. Man findet in den Pusteln Staphylococcus pyogenes aureus, albus, citreus, cereus. Die Sykosis ist eine Folliculitis der dicht be haarten Hautgegenden; die Folliculitis findet sich an wenig behaarten Stellen. E. beobachtete einen $\delta$ Ojährigen Mann, der seit 7 Jahren an wiederkehrenden Entzündungen im Nacken litt, die nach einem Antrax aufgetreten. Die ganze Haut des Nackens gerötet, bretthart, schmerzhaft; auf Druck entleert sich aus vielen Eiterpunkten Eiter mit zahlreichon Staphylokken. Zunächst Auflage einer 15 procentigen Resorcinpasta, dann kataphoretische Behandlung · mit Ichtyollösung, worauf sich die Eiteröffnungen bis auf eine Stelle links von der Mittellinie des Nackens schlossen. Híer waren mehrere Mündungen, aus denen. zum Teil Haarbüscbelchen ragten und Jahre lang sich Fiter entleerte. Zum Teil standen die Oeffnungen durch Fisteln unter der Oberfläche mit einander in Verbindung. Der Patient musste statt des steifleinenen Hemdkragens tiefsitzende weiche Seidenhemden tragen. Die Kataphorese führte E. so aus: In ein glockenförmiges Glasgefäss von 5-6 cm Durchmesser ragt bis zur Mítte der Höhe ein Stift aus amalgamiertem Zink mit kleiner Platte, der mit einer Batterie verbunden ist. Die Glocke wird mit Bruns'scher Watte gefiillt, auf weiche 20-30procentige Ichthyollösung gegossen ist. Die Kathode wird schnell auf die Haut angedrückt, die Anode befindet sich auf beliebiger Körperstelle; ein Strom von $2 \mathrm{u}$ M.-A. wird durchgeleitet. Nur wenn der Process in den Follikeln und oberflächlicher gelegen, hat diese Behandlung Erfolg. Liegt er tiefer, so sind tiefe Incisionen am Platze. Ausserdem sind die Brutstätten der Staphylokokken zu vernichten; Nasenhöhle, Gehörgang, Mundhöhle, Genitalion müssen gereinigt, die Haarpflege geordnet werden. GíOrge Meyer (Berlin).

Die Diät bei Hautkrankheiten, weiche in den Vorstellungen der Laien-welt eine so tief greifende Roilo spielt. dass man fast versucht sein möchte, einer etwa greifbaren Unterlage derselben nachzuspüren, diirfte auch in der wissen-schaftlichen Medicin vielleicht wieder mehr an Boden gewinnon. Die Fleísch-Entziehungscur bei Psoriasis giebt hierauf bereits einen Hinweis. Es ist bekannt. dass, wenn man Psoriatikern, ohne sie auf vregetabilische Kost zu setzen, ihnen vielmehr Fische, Eier, Butter, Milch neben sonst reichlicher und stickstoffhaltiger Nahrung reicht, dabei aber das Fleisch der Säugetíere und Vögel ganz verbietet. die Behandlung des Hautleidens mit sonst iiblichen Methoden eine erkennbare Unterstützung findet. Auch wird berichtet, dass diese Lebensweise allein schon die Psoriasis giinstig beoinílusse. Ich ka nn das im Ganzen bestiitigen, doch ist die Wirksamkeit dieser Diätform nur eine untergeordnete. Ganz abgesehen davon. dass sie sich auf die Dauer ohne grusse Störungen nicht durchführen lässt, bleibt ihr Einrluss doch nicht kräftig oder weittragend genug, um ihn practisch all-gemeiner zu verwerten. Aber in thooretischem Sinn mag diese Erfahrung über den Zusammenhang zwischen einer äusserlichen Haftung wie Psoriasis, und der Emährungsweise der Patienten immerhin als ein Hinweis gelten. Allerdings fehlt einstweilen noch jede plausible Erklärung. - Umgekehrt bin ich seit 
einiger Zeit bei der $\Delta$ llgemein-Behandlung der Furunkulose von einer bestimmten Hypothese ausgegangen und zwar, wie hervorgehoben werden darf, nicht ganz ohne sichtlichen Erfolg. Niemand, so niusste ich mir sagen, neigt mehr zu Furunkeln und Carbunkeln, als der Diabetiker. Sobald aber entsprechende Diät den Zuckergehalt seiner $\mathrm{G}^{1} / 8$ webssäfte eliminirt hat, erlischt diese Disposition in prompter Weise. Da nun tür das Entstehen jener Art von Eiternecrosen lediglich die Anwesenheit von Staphylokokken verantwortlich gemacht werden darf, so kann man nicht anders als annehmen, dass sie im zuckerhaltigen Gewebe einen sehr begiinstigten, im zuckerarmen aber einen verhältnismässig sterilen Nährboden fl·nden. Dementsprechend habe ich auch solchen Furunkel - Kranken, welche ohne jede Melliturie, an ihren Beschwerden seit Monaten und Jahren gelitten batten, eine gleiche Lebensweise auferlegt, wie man sie Zuckerkranken vorschreibt: Gebrauch von Karlsbader Mühlbrunnen, stricte Untersagung von Zucker und Amylaceen. Diese Schaffung eines Zuckerliungers hat die von mir seither beobachteten Furunkulosen in so giinstiger Weise gehemmt, dass sie tür eine diätetische Behandlung von Kranken solcher Art immerhin angeführt werden darf. Lassar.

Eine neue Art Salbe zu dosireii, geht von der Actien-Gesellschaft für Anilin-Fabrikation in Berlin aus. Dieselbe vertreibt ihre Resorbin-Hg. Salbe in tarirten Glascylindern. Ein Stempel sehiebt den Inhalt laut angebrachter Scala grammweise heraus. Der zurückbleibende Rest ist vor jeder Verunreinigung ge-schützt und das Verfahren muss als originell und praktisch bezoichnet werden.

$\mathrm{Zu}$ der vom ]4.-16. Mai d. J. in Breslau stattfmdenden I $\Lambda$ r. Congress der Deutschen üermatologischen Gesellsehaft sind bisher folgende Referate, Vorträge und Demo $\pi$ strationen angemeldet: Haupt-themata: 1. Die modernen Systematisirungsversuche in der Dermatologie. Referent: Kaposi (Wien). Diskussion: Schwimmer (Pest). 2. Der augen-blickliche Stand der Dermatomycosenlehre. Referent: Pick (Prag). Demonstration von Culturen: Winternitz, Kroesing. - Perner sind angemeldet: ^orträge und Demonstrationen über Gronorrhoe, Endoskopie etc. von Grünfeld, Jacobi, Jadassobn, Kocb, Kollmann, Loewenhardt, Lohn-stein, A. Neisser, Putzler, Scbäffer, Steinschneider etc. - Dermatologische $\Lambda$ Torträge: Caspary (Erythema exsudativum), Doutrelepont (Zur Haut-tuberculose), Friedheim (Einwirkung von Säuren auf die Haut), van Hoorn (Thema vorbehalten), Joseph (Ungewöhnliche Ichthyosisformen), Lasch (Urticaria factitia), Ledermann (Resorbin), Lesser (Herpes Zoster), Mracek (Aetiologie der toxischen Erytheme), Neuberger (Lichen ruber), Riebl (Hauttuberculose), Rosentbal (Blasenbildende Affectionen der Mundschleim-haut), Saalfeld (Phaneroscopie und Glasdruck), Schwimmer (Hysterische Hautgangraen), von Sehlen (Eczem und Schleimhauterkrankung), Staub (Erythromelalgie, Therapie der Hautactinomycose), Winternitz (Allgemein-wirkung hautreizender Stofle). - Syphilidologiscbe $\Lambda$ rorträge und De-monstrationen: Block (Bubonenbehandlung), Lues und Tuberculose (Hoch-singer, Jadassohn, A. Xeisser), Marschalko (Spätlues), J. Neumann (Syphilis der Speicheldrüsen). - Dermatologische Vorträge mit Demonstrationen: Arning (Lepra), Dreysel und Oppler (Eleidin), Ehrmann (Lymph-gefässe der männlichen Genitalien), Pabry (Urticaria pigmentosa), Galewski (Lepröse Trophoneurose), Halle (Hautmodelle), Kroesing (Zur Lapus-behandlung), Mikulicz (Angiombehandlung), Münchheimer (Herpes Zoster), A. Neisser (Molluscum contagiosum), Ruffer (CarcinomPsorospermien), Touton (Mollnscum contagiosum). - Krankendemonstrationen von Chotzen, Jadassohn, A. Neisser. 